\title{
Comparison Theorems of Spectral Radius for Splittings of Matrices
}

\author{
Cui-Xia $\mathrm{Li}^{1}$ and $\mathrm{Su}-\mathrm{Hua} \mathrm{Li}^{2}$ \\ ${ }^{1}$ School of Mathematics and Statistics, Anyang Normal University, Anyang 455000, China \\ ${ }^{2}$ School of Mathematics and Statistics, Yunnan University, Kunming 650091, China \\ Correspondence should be addressed to Cui-Xia Li; lixiatk@126.com \\ Received 12 May 2014; Accepted 27 June 2014; Published 8 July 2014 \\ Academic Editor: Shuqian Shen \\ Copyright (C) 2014 C.-X. Li and S.-H. Li. This is an open access article distributed under the Creative Commons Attribution License, \\ which permits unrestricted use, distribution, and reproduction in any medium, provided the original work is properly cited. \\ A class of the iteration method from the double splitting of coefficient matrix for solving the linear system is further investigated. \\ By structuring a new matrix, the iteration matrix of the corresponding double splitting iteration method is presented. On the basis \\ of convergence and comparison theorems for single splittings, we present some new convergence and comparison theorems on \\ spectral radius for splittings of matrices.
}

\section{Introduction}

Let us consider the following linear system:

$$
A x=b,
$$

where $A \in \mathbb{R}^{n \times n}$ is a nonsingular matrix, $b \in \mathbb{R}^{n \times 1}$ is a given vector, and $x \in \mathbb{R}^{n \times 1}$ is an unknown vector. In order to solve the linear system (1) by iteration methods, the coefficient matrix $A$ is split into

$$
A=M-N
$$

where $M$ is nonsingular; then, an iterative formula for solving the linear system (1) is

$$
\begin{array}{r}
x^{k+1}=M^{-1} N x^{k}+M^{-1} b \equiv T x^{k}+M^{-1} b, \\
k=0,1,2, \ldots,
\end{array}
$$

where $T=M^{-1} N$ is the iteration matrix in (3).

The splitting (2) is called a (single) splitting of $A$ and the iteration method (3) is called a (one-step) linear stationary iteration method. Obviously, the iteration method (3) converges to the unique solution of the linear system (1) if and only if the spectral radius $\rho(T)$ of the iteration matrix $T$ is smaller than 1 . The spectral radius of the iteration matrix is decisive for the convergence and stability, and the smaller it is, the faster the iteration method converges when the spectral radius is smaller than 1 . So far, many comparison theorems of single splittings of matrices have been presented in some papers and books [1-8].

Woźnicki [9] introduced the double splitting of $A$ as

$$
A=P-R-S
$$

where $P$ is a nonsingular matrix. The corresponding iterative scheme is spanned by three successive iterations:

$$
\begin{array}{r}
x^{k+1}=P^{-1} R x^{k}+P^{-1} S x^{k-1}+P^{-1} b, \\
k=0,1,2, \ldots,
\end{array}
$$

which can be rewritten in the equivalent form

$$
\left[\begin{array}{c}
x^{k+1} \\
x^{k}
\end{array}\right]=\left[\begin{array}{cc}
P^{-1} R & P^{-1} S \\
I & 0
\end{array}\right]\left[\begin{array}{c}
x^{k} \\
x^{k-1}
\end{array}\right]+\left[\begin{array}{c}
P^{-1} b \\
0
\end{array}\right],
$$

where $I$ is the identity matrix. The iteration method given by (6) converges to the unique solution of (1) for all initial vectors $x^{0}, x^{1}$ if and only if the spectral radius of the iteration matrix

$$
W=\left[\begin{array}{cc}
P^{-1} R & P^{-1} S \\
I & 0
\end{array}\right]
$$

is less than one; that is, $\rho(W)<1$. 
Recently, some convergence and comparison results for double splittings of matrices are presented. In [10], some convergence theorems for the double splitting of a monotone matrix or a Hermitian positive definite matrix are presented. Compared with the results in [10], some improved convergence and comparison results for the double splitting of a Hermitian positive definite matrix are proposed in [11]. In [12], some convergence results for the double splitting of a non-Hermitian positive semidefinite matrix are established. Further, some comparison theorems for double splittings of different monotone matrices are given in $[13,14]$ and some convergence and comparison results for nonnegative double splittings of matrices are given in $[4,15]$. In this paper, by structuring a new matrix, the iteration matrix of the corresponding iteration method from double splitting of coefficient matrix is presented. On the basis of convergence and comparison theorems for single splittings, we present some new convergence and comparison theorems on spectral radius for splittings of matrices.

\section{Preliminaries}

For convenience, we give some notations, definitions, and lemmas which will be used in the sequel.

The matrix $A$ is called nonnegative and is denoted by $A \geq$ 0 if $a_{i j} \geq 0$ for $i, j=1,2, \ldots, n$. We write $A \geq B(A>B)$ if $a_{i j} \geq b_{i j}\left(a_{i j}>b_{i j}\right)$ for $i, j=1,2, \ldots, n$. The matrix $A$ is called a monotone matrix if $A^{-1} \geq 0$.

Definition 1. Let $A$ be a nonsingular matrix. Then, $A=M-N$ is called

(i) regular if $M^{-1} \geq 0$ and $N \geq 0$;

(ii) weak regular if $M^{-1} \geq 0$ and $M^{-1} N \geq 0$;

(iii) nonnegative if $M^{-1} N \geq 0$;

(iv) $M$-splitting if $M$ is an $M$-matrix and $N \geq 0$.

Definition 2 (see $[4,10,15])$. Let $A$ be a nonsingular matrix. Then, the double splitting $A=P-R-S$ is

(i) convergent if and only if $\rho(W)<1$;

(ii) a regular double splitting if $P^{-1} \geq 0, R \geq 0$, and $S \geq 0$;

(iii) a weak regular double splitting if $P^{-1} \geq 0, P^{-1} R \geq 0$, and $P^{-1} S \geq 0$;

(iv) a nonnegative splitting if $P^{-1} R \geq 0$ and $P^{-1} S \geq 0$;

(v) an $M$-double splitting if $P$ is an $M$-matrix and $R \geq 0$ and $S \geq 0$.

Lemma 3 (see [2]). Let $A \geq 0$. Then

$$
\begin{aligned}
& \alpha x \leq A x, \quad x \geq 0, \quad \text { implies } \alpha \leq \rho(A), \\
& A x \leq \beta x, \quad x>0, \quad \text { implies } \rho(A) \leq \beta .
\end{aligned}
$$

Lemma 4 (see [16]). Let $A \in \mathbb{R}^{n \times n}$ and $A=M_{1}-N_{1}=$ $M_{2}-N_{2}$ be $M$-splittings of A (i.e., $M_{i}$ are $M$-matrices; $N_{i} \geq 0$, $i=1,2)$ and

$$
N_{1} \geq N_{2}, \quad N_{1} \neq N_{2}, \quad N_{2} \neq 0 .
$$

Then, exactly one of the following statements holds:

(1) $0 \leq \rho\left(M_{2}^{-1} N_{2}\right)<\rho\left(M_{1}^{-1} N_{1}\right)<1$. In addition, if $A$ is irreducible, the first inequality is also strict;

(2) $\rho\left(M_{2}^{-1} N_{2}\right)=\rho\left(M_{1}^{-1} N_{1}\right)=1$;

(3) $\rho\left(M_{2}^{-1} N_{2}\right)>\rho\left(M_{1}^{-1} N_{1}\right)>1$.

Lemma 5 (see [17]). Let $A \in \mathbb{R}^{n \times n}$ and $A=M_{1}-N_{1}=M_{2}-$ $\mathrm{N}_{2}$ be nonnegative and convergent.

(1) If either $M_{1}^{-1} M_{2} \geq$ I or $M_{2}^{-1} M_{1} \leq I$, then $\rho\left(M_{1}^{-1} N_{1}\right)<$ $\rho\left(M_{2}^{-1} N_{2}\right)<1$.

(2) If there exist $\alpha, 0<\alpha<1$, such that $M_{1}^{-1} M_{2} \geq(1 / \alpha) I$ or $M_{2}^{-1} M_{1} \leq \alpha I$, then $\rho\left(M_{1}^{-1} N_{1}\right)<\rho\left(M_{2}^{-1} N_{2}\right)<1$.

\section{Comparison Theorems}

Let

$$
A=P_{1}-R_{1}-S_{1}=P_{2}-R_{2}-S_{2},
$$

be two double splittings of $A$. Then, we define

$$
W_{1}=\left[\begin{array}{cc}
P_{1}^{-1} R_{1} & P_{1}^{-1} S_{1} \\
I & 0
\end{array}\right], \quad W_{2}=\left[\begin{array}{cc}
P_{2}^{-1} R_{2} & P_{2}^{-1} S_{2} \\
I & 0
\end{array}\right] .
$$

Let

$$
\mathbb{A}=\left[\begin{array}{cc}
A & 0 \\
-I & I
\end{array}\right]
$$

Then, $\mathbb{A} \in \mathbb{R}^{2 n \times 2 n}$ and

$$
\mathbb{A}^{-1}=\left[\begin{array}{ll}
A^{-1} & 0 \\
A^{-1} & I
\end{array}\right] .
$$

This shows that $\mathbb{A}$ is nonsingular whenever $A$ is nonsingular. Let $\mathbb{A}$ be split as

$$
\mathbb{A}=\mathbb{M}-\mathbb{N}
$$

with

$$
\mathbb{M}=\left[\begin{array}{ll}
P & S \\
0 & I
\end{array}\right], \quad \mathbb{N}=\left[\begin{array}{cc}
R+S & S \\
I & 0
\end{array}\right]
$$

Then

$$
W=\mathbb{M}^{-1} \mathbb{N}=\left[\begin{array}{cc}
P^{-1} R & P^{-1} S \\
I & 0
\end{array}\right] .
$$

In [4], some comparison theorems for the double splitting (4) through investigating the matrix splitting defined by (14) were obtained, which were described as follows.

Theorem 6 (see [4]). Let $A^{-1} \geq 0$, and let the two double splittings (10) be nonnegative and convergent. Suppose

$$
P_{1} \leq P_{2}, \quad S_{1} \leq S_{2}
$$


then

$$
\rho\left(W_{1}\right) \leq \rho\left(W_{2}\right)
$$

Corollary 7 (see [4]). Let $A^{-1} \geq 0$, and let the two double splittings (10) be nonnegative and convergent. Suppose

$$
R_{1} \leq R_{2}, \quad S_{1} \leq S_{2}
$$

then

$$
\rho\left(W_{1}\right) \leq \rho\left(W_{2}\right)
$$

Theorem 8 (see [4]). Let $A^{-1} \geq 0, A=P_{1}-R_{1}-S_{1}$ be regular double splitting, and let $A=P_{2}-R_{2}-S_{2}$ be nonnegative and convergent double splitting. Suppose

$$
P_{1}^{-1} \geq P_{2}^{-1}, \quad P_{1}^{-1} S_{1} \leq P_{2}^{-1} S_{2}
$$

then $\rho\left(W_{1}\right) \leq \rho\left(W_{2}\right)$.

In [4], they claimed that $\mathbb{A}$ is nonsingular whenever $A$ is nonsingular. In fact, we make use of the following strategy to make $\mathbb{A}$ nonsingular. That is to say,

$$
\mathbb{M}^{-1} \mathbb{A}=I-\mathbb{M}^{-1} \mathbb{N}
$$

Obviously, if $I-\mathbb{M}^{-1} \mathbb{N}$ is nonsingular, then we immediately obtain that matrix $\mathbb{A}$ is nonsingular too. When one discusses the convergence properties of the iteration scheme (6), it is expected that the spectral radius $\rho(W)$ of the iteration matrix $W=\mathbb{M}^{-1} \mathbb{N}$ is less than one. In this case, the iteration scheme (6) is convergent. In this meanwhile, we also know that $\mathbb{A}$ is nonsingular. Further, comparison theorems discussed are more meaningful as the spectral radius $\rho(W)$ of the iteration matrix $W=\mathbb{M}^{-1} \mathbb{N}$ is less than one. Based on this idea, we can consider the choice of matrixes $\mathbb{M}$ and $\mathbb{N}$ as

$$
\mathbb{M}=\left[\begin{array}{ll}
P & 0 \\
0 & I
\end{array}\right], \quad \mathbb{N}=\left[\begin{array}{ll}
R & S \\
I & 0
\end{array}\right]
$$

In light of this choice, we also have the same as the iteration matrix $W$,

$$
W=\mathbb{M}^{-1} \mathbb{N}=\left[\begin{array}{cc}
P^{-1} R & P^{-1} S \\
I & 0
\end{array}\right] .
$$

In this case, the matrix $\mathbb{A}$ is not

$$
\mathbb{A}=\left[\begin{array}{cc}
A & 0 \\
-I & I
\end{array}\right]
$$

but is

$$
\mathbb{A}=\left[\begin{array}{cc}
P-R & -S \\
-I & I
\end{array}\right]
$$

Then, we have

$$
\mathbb{A}=\mathbb{M}-\mathbb{N} \equiv\left[\begin{array}{ll}
P & 0 \\
0 & I
\end{array}\right]-\left[\begin{array}{ll}
R & S \\
I & 0
\end{array}\right] .
$$

Based on Lemma 4, we have the following results.

Theorem 9. Let

$$
\mathbb{A}=\mathbb{M}_{i}-\mathbb{N}_{i}=\left[\begin{array}{cc}
P_{i} & 0 \\
0 & I
\end{array}\right]-\left[\begin{array}{cc}
R_{i} & S \\
I & 0
\end{array}\right] \quad(i=1,2),
$$

be $M$-splittings of $\mathbb{A}$. If $R_{1} \leq R_{2}$, then exactly one of the following statements holds:

(1) $0 \leq \rho\left(W_{1}\right)<\rho\left(W_{2}\right)<1$. In addition, if $\mathbb{A}$ is irreducible, the first inequality is also strict;

(2) $\rho\left(W_{1}\right)=\rho\left(W_{2}\right)=1$;

(3) $\rho\left(W_{1}\right)>\rho\left(W_{2}\right)>1$.

Proof. For $i=1,2$, let

$$
\mathbb{M}_{i}=\left[\begin{array}{cc}
P_{i} & 0 \\
0 & I
\end{array}\right], \quad \mathbb{N}_{i}=\left[\begin{array}{cc}
R_{i} & S \\
0 & I
\end{array}\right] .
$$

Then

$$
\mathbb{A}=\mathbb{M}_{i}-\mathbb{N}_{i}, \quad W_{i}=\mathbb{M}_{i}^{-1} \mathbb{N}_{i}
$$

Since $R_{1} \leq R_{2}$, then $\mathbb{N}_{1} \leq \mathbb{N}_{2}$. That is, from Lemma 4 , the results in Theorem 9 hold true.

Theorem 10. Let

$$
\mathbb{A}=\mathbb{M}_{i}-\mathbb{N}_{i}=\left[\begin{array}{cc}
P_{i} & 0 \\
0 & I
\end{array}\right]-\left[\begin{array}{cc}
R_{i} & S \\
I & 0
\end{array}\right] \quad(i=1,2),
$$

be nonnegative and convergent. If either $P_{1}^{-1} P_{2} \geq I$ or $P_{2}^{-1} P_{1} \leq$ $I$, then $\rho\left(W_{1}\right)<\rho\left(W_{2}\right)<1$.

Proof. Let

$$
\mathbb{A}=\mathbb{M}_{i}-\mathbb{N}_{i}=\left[\begin{array}{cc}
P_{i} & 0 \\
0 & I
\end{array}\right]-\left[\begin{array}{cc}
R_{i} & S \\
I & 0
\end{array}\right] \quad(i=1,2),
$$

be nonnegative. By direct operation, we obtain

$$
\mathbb{M}_{1}^{-1} \mathbb{M}_{2}=\left[\begin{array}{cc}
P_{1}^{-1} P_{2} & 0 \\
0 & I
\end{array}\right], \quad \mathbb{M}_{2}^{-1} \mathbb{M}_{1}=\left[\begin{array}{cc}
P_{2}^{-1} P_{1} & 0 \\
0 & I
\end{array}\right] \text {. }
$$

Since $P_{1}^{-1} P_{2} \geq I$ or $P_{2}^{-1} P_{1} \leq I$, we have $\mathbb{M}_{1}^{-1} \mathbb{M}_{2} \geq I$ or $\mathbb{M}_{2}^{-1} \mathbb{M}_{1} \leq I$. From Lemma 5 , the results of Theorem 10 hold true.

Obviously, from Lemma 5, we have the following result.

Theorem 11. Let

$$
\mathbb{A}=\mathbb{M}_{i}-\mathbb{N}_{i}=\left[\begin{array}{cc}
P_{i} & 0 \\
0 & I
\end{array}\right]-\left[\begin{array}{cc}
R_{i} & S \\
I & 0
\end{array}\right] \quad(i=1,2),
$$

be nonnegative and convergent. If there exist $\alpha, 0<\alpha<1$, such that $P_{1}^{-1} P_{2} \geq(1 / \alpha) I$ or $P_{2}^{-1} P_{1} \leq \alpha I$, then $\rho\left(W_{1}\right)<\rho\left(W_{2}\right)<1$. 
Compared with Corollary 7 and Theorem 8, the condition $A^{-1} \geq 0$ in Theorems 9,10 , and 11 is not necessary.

Theorem 12. Let

$$
\begin{gathered}
\mathbb{A}^{-1}=\left[\begin{array}{cc}
P-R & -S \\
-I & I
\end{array}\right]^{-1} \geq 0, \\
\mathbb{A}=\mathbb{M}_{i}-\mathbb{N}_{i}=\left[\begin{array}{cc}
P_{i} & 0 \\
0 & I
\end{array}\right]-\left[\begin{array}{cc}
R_{i} & S \\
I & 0
\end{array}\right] \quad(i=1,2),
\end{gathered}
$$

be nonnegative and convergent. If $P_{1} \leq P_{2}$, then $\rho\left(W_{1}\right)<$ $\rho\left(W_{2}\right)<1$.

Proof. Obviously, if $P_{1} \leq P_{2}$, then $\mathbb{M}_{1} \leq \mathbb{M}_{2}$. From Theorem 2.11 in [17], the results of Theorem 12 hold true.

Theorem 13. Let

$$
\mathbb{A}^{-1}=\left[\begin{array}{cc}
P-R & -S \\
-I & I
\end{array}\right]^{-1} \geq 0,
$$

let $\mathbb{A}=\mathbb{M}_{1}-\mathbb{N}_{1}$ be regular, and let $\mathbb{A}=\mathbb{M}_{2}-\mathbb{N}_{2}$ be nonnegative and convergent. If $P_{2}^{-1} \leq P_{1}^{-1}$, then $\rho\left(W_{1}\right)<\rho\left(W_{2}\right)<1$.

Proof. Assume that $\rho\left(W_{1}\right)=0$; the result is trivial. Assume that $\rho\left(W_{1}\right)>0$. Then, the splitting $\mathbb{A}=\mathbb{M}_{1}-\mathbb{N}_{1}=\mathbb{M}_{2}-\mathbb{N}_{2}$ is nonnegative.

By the Perron-Frobenius Theorem in [1], there exists a vector $x \geq 0$ and $x \neq 0$, such that $W_{1} x=\rho\left(W_{1}\right) x$. From $\mathbb{N}_{1} \geq 0$, we derive $\mathbb{N}_{1} x \geq 0$. Since $P_{2}^{-1} \leq P_{1}^{-1}$, then $\mathbb{M}_{2}^{-1} \leq \mathbb{M}_{1}^{-1}$. From Theorem 2.17 in [17], the results of Theorem 13 hold true.

\section{Numerical Example}

In this section, we make use of an example to illustrate Theorems 9, 10, 12, and 13 .

Example 1. Assume that

$$
\mathbb{A}=\left[\begin{array}{cccc}
3 & 0 & -1 & -1 \\
0 & 4 & -1 & -1 \\
-1 & 0 & 1 & 0 \\
0 & -1 & 0 & 1
\end{array}\right]
$$

Then

$$
\mathbb{A}^{-1}=\left[\begin{array}{llll}
0.6 & 0.2 & 0.8 & 0.8 \\
0.2 & 0.4 & 0.6 & 0.6 \\
0.6 & 0.2 & 1.8 & 0.8 \\
0.2 & 0.4 & 0.6 & 1.6
\end{array}\right] \geq 0 .
$$

Let

$$
\begin{array}{rlrl}
\mathbb{M}_{1} & =\left[\begin{array}{llll}
4 & 0 & 0 & 0 \\
0 & 5 & 0 & 0 \\
0 & 0 & 1 & 0 \\
0 & 0 & 0 & 1
\end{array}\right], & \mathbb{N}_{1}=\left[\begin{array}{cccc}
1 & 0 & -1 & -1 \\
0 & 1 & -1 & -1 \\
1 & 0 & 0 & 0 \\
0 & 1 & 0 & 0
\end{array}\right], \\
\mathbb{M}_{2}=\left[\begin{array}{llll}
5 & 0 & 0 & 0 \\
0 & 6 & 0 & 0 \\
0 & 0 & 1 & 0 \\
0 & 0 & 0 & 1
\end{array}\right], & \mathbb{N}_{2}=\left[\begin{array}{cccc}
2 & 0 & -1 & -1 \\
0 & 2 & -1 & -1 \\
1 & 0 & 0 & 0 \\
0 & 1 & 0 & 0
\end{array}\right] .
\end{array}
$$

Then

$$
P_{1}^{-1}=\left[\begin{array}{cc}
\frac{1}{4} & 0 \\
0 & \frac{1}{5}
\end{array}\right], \quad P_{2}^{-1}=\left[\begin{array}{cc}
\frac{1}{5} & 0 \\
0 & \frac{1}{6}
\end{array}\right]
$$

Therefore, we have the following facts. That is to say,

$$
\begin{gathered}
R_{1} \leq R_{2} ; \quad P_{1}^{-1} P_{2} \geq I, \quad P_{2}^{-1} P_{1} \leq I \\
P_{1} \leq P_{2} ; \quad P_{2}^{-1} \leq P_{1}^{-1}
\end{gathered}
$$

which, respectively, satisfy the conditions of Theorems 9, 10, 12 , and 13 . In this case, by the simple computations, we have

$$
\rho\left(W_{1}\right)=0.7949, \quad \rho\left(W_{2}\right)=0.8195 \text {. }
$$

Clearly, $\rho\left(W_{1}\right) \leq \rho\left(W_{2}\right)<1$. That is to say, Theorems $9,10,12$, and 13 hold true.

\section{Conflict of Interests}

The authors declare that there is no conflict of interests regarding the publication of this paper.

\section{Acknowledgments}

This research was supported by NSFC (no. 11301009), by Science \& Technology Development Plan of Henan Province (no. 122300410316), and by Natural Science Foundations of Henan Province (no. 13A110022).

\section{References}

[1] R. S. Varga, Matrix Iterative Analysis, Springer, Berlin, Germany, 2000.

[2] A. Berman and R. J. Plemons, Nonnegative Matrices in the Mathematics Sciences, SIAM, Philadelphia, Pa, USA, 1994.

[3] D. M. Young, Iterative Solution of Large Linear Systems, Academic Press, New York, NY, USA, 1971.

[4] J. Song and Y. Song, "Convergence for nonnegative double splittings of matrices," Calcolo, vol. 48, no. 3, pp. 245-260, 2011.

[5] Z. I. Woźnicki, "Nonnegative splitting theory," Japan Journal of Industrial and Applied Mathematics, vol. 11, no. 2, pp. 289-342, 1994.

[6] Z. I. Woźnicki, "Basic comparison theorems for weak and weaker matrix splittings," Electronic Journal of Linear Algebra, vol. 8, pp. 53-59, 2001.

[7] M. Benzi and D. B. Szyld, "Existence and uniqueness of splittings for stationary iterative methods with applications to alternating methods," Numerische Mathematik, vol. 76, no. 3, pp. 309-321, 1997.

[8] L. Elsner, A. Frommer, R. Nabben, and D. B. Szyld, "Conditions for strict inequality in comparisons of spectral radii of splittings of different matrices," Linear Algebra and Its Applications, vol. 363, pp. 65-80, 2003.

[9] Z. I. Woźnicki, "Estimation of the optimum relaxation factors in partial factorization iterative methods," SIAM Journal on Matrix Analysis and Applications, vol. 14, no. 1, pp. 59-73, 1993. 
[10] S.-Q. Shen and T.-Z. Huang, "Convergence and comparison theorems for double splittings of matrices," Computers \& Mathematics with Applications, vol. 51, no. 12, pp. 1751-1760, 2006.

[11] S.-Q. Shen, T.-Z. Huang, and J.-L. Shao, "Convergence and comparison results for double splittings of Hermitian positive definite matrices," Calcolo, vol. 44, no. 3, pp. 127-135, 2007.

[12] C. Zhang, "On convergence of double splitting methods for non-Hermitian positive semidefinite linear systems," Calcolo, vol. 47, no. 2, pp. 103-112, 2010.

[13] S.-X. Miao and B. Zheng, "A note on double splittings of different monotone matrices," Calcolo, vol. 46, no. 4, pp. 261266, 2009.

[14] C. Li, Q. Cui, and S. Wu, "Comparison theorems for single and double splittings of matrices," Journal of Applied Mathematics, vol. 2013, Article ID 827826, 4 pages, 2013.

[15] C. Li and S. Wu, "Some new comparison theorems for double splittings of matrices," Applied Mathematics \& Information Sciences, vol. 8, no. 5, pp. 2523-2526, 2014.

[16] W. Li, L. Elsner, and L. Lu, "Comparisons of spectral radii and the theorem of Stein-Rosenberg," Linear Algebra and its Applications, vol. 348, pp. 283-287, 2002.

[17] Y. Z. Song, "Comparison theorems for splittings of matrices," Numerische Mathematik, vol. 92, no. 3, pp. 563-591, 2002. 


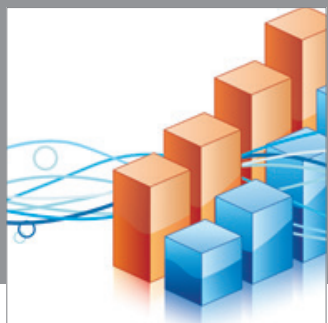

Advances in

Operations Research

mansans

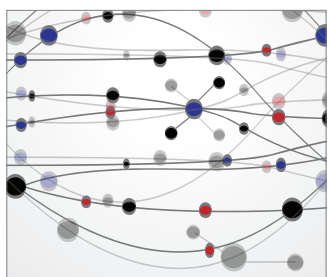

The Scientific World Journal
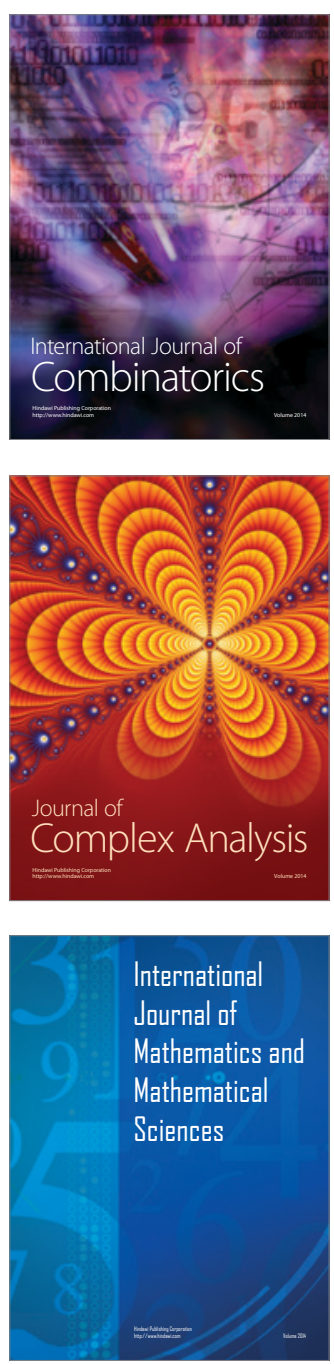
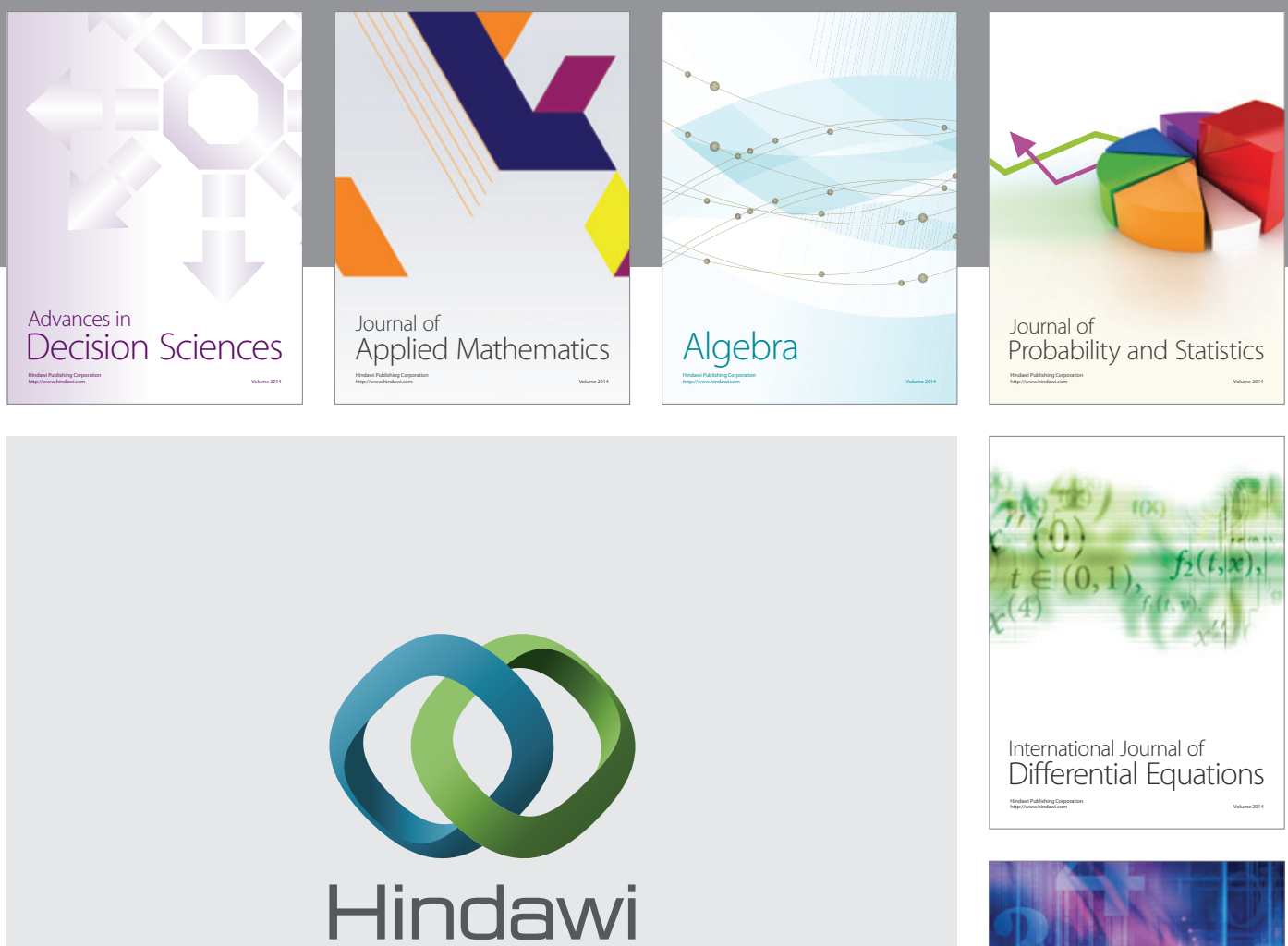

Submit your manuscripts at http://www.hindawi.com
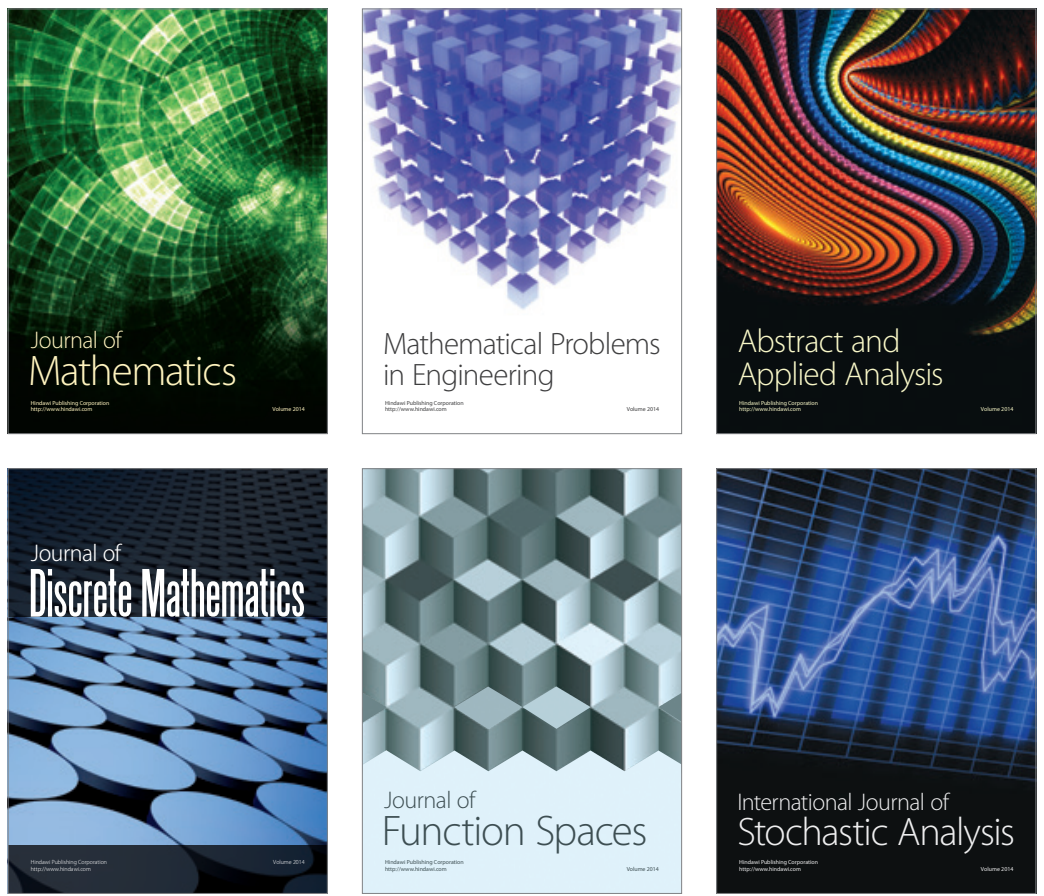

Journal of

Function Spaces

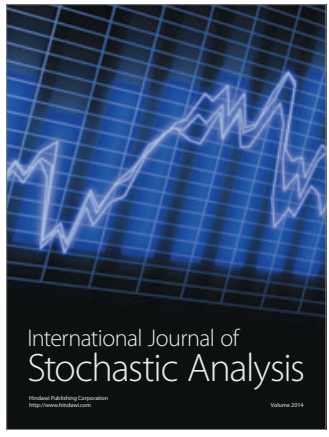

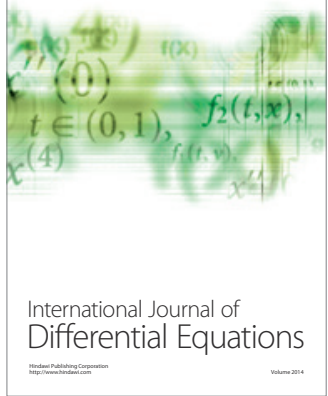
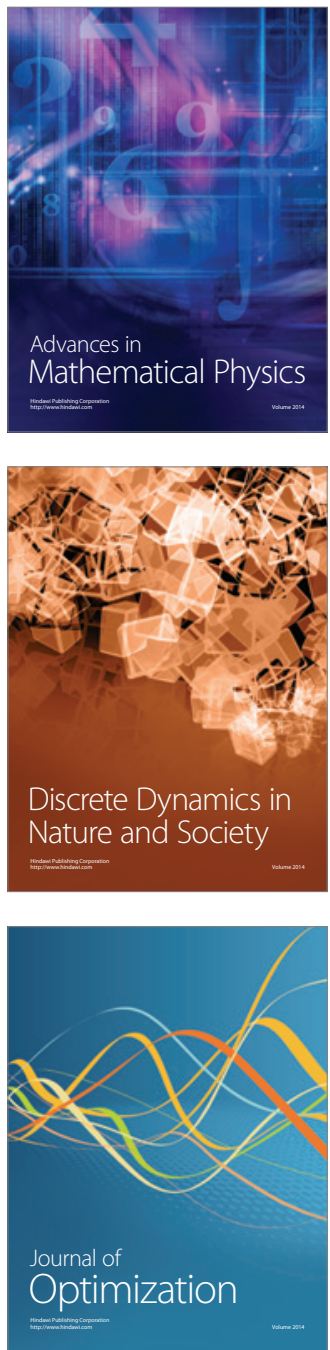